\title{
Cardiovascular Collapse during Induction of General Anesthesia
}

\author{
Pradhan $B^{1}$ \\ ${ }^{1}$ Insatitute of Medicine, Maharajgunj, Kathmandu, Nepal.
}

\begin{abstract}
Anaphylactic reactions to anesthetic drugs, though rare, may be encountered by anyone involved. The clinical manifestation of the anaphylactic reactions, which may be different from patient to patient, is caused by the histamine released either from the mast cells or basophils or both. Laboratory investigations can determine the source of histamine release. Here, I present a case of anaphylactic reaction to sodium thiopental. The main stay of the treatment is oxygenation, fluids and epinephrine, which was applied in this case also, with successful outcome of the patient.
\end{abstract}

Key words: Adrenaline, Anaphylaxis, Anaphylactoid, Collapse, Thiopentole.

\section{INTRODUCTION}

Anaphylactoid reactions are more common than the true anaphylactic reactions with drugs related to anesthesia. Cardiovascular collapse presented with hypotension and tachycardia are the most common presentation of anaphylactoid reactions. Here I present one case of cardiovascular collapse following anesthetic drug administration.

\section{CASE REPORT}

A 22 years old male, weighing $60 \mathrm{~kg}$ with the diagnosis of left chronic suppurative otitis media tubo tympany was planned for elective left modified radical mastoidectomy. In preanesthetic check up the patient did not give any history of previous exposure to anesthetia, drug allergy or any other systemic pathology. Investigations were within normal limits. Heart rate was 72 per minute regular, blood pressure $110 / 80 \mathrm{~mm}$ of
$\mathrm{Hg}$ in general physical examination. Systemic examinations were also normal. Airway evaluation revealed the patient of Mallampati Class II with free temporomandibular and atlanto occipital joints range of motion. The patient was categorized as of ASA I class. General anesthesia was planned for the patient. The patient was premedicated with Diazepam $10 \mathrm{mg}$ orally night before surgery and in the morning of surgery.

In the operation theatre on the day of surgery, monitors attached were pulse oximetry, electrocardiogram (ECG) and blood pressure. Intravenous (IV) line was opened in left hand with $18 \mathrm{G}$ cannula. Preoperative blood pressure was $110 / 80 \mathrm{~mm}$ of $\mathrm{Hg}$, pulse $80 / \mathrm{min}$ regular. Oxygen saturation was $99 \%$ in room air. Pethidine $40 \mathrm{mg}$ was given and after 5 minutes anesthesia was induced with Sodium thiopentone $5 \mathrm{mg} / \mathrm{kg}$. Immediate fall in oxygen saturation was noticed upto $90 \%$ which further decreased to $80 \%$ despite $100 \%$ oxygenation and manual ventilation,

\author{
Correspondence: \\ Dr. Bishwas Pradhan \\ Department of Anesthesiology \\ IOM, Maharajguni, Kathmandu, Nepal. \\ E mail: biswaspradhan@yahoo.com
}


Pradhan. Cardiovascular Collapse during Induction of General Anesthesia - A Case Report.

that was started after apnoea. Generalised sweating all over the body was noticed with predominance over the facial region. The patient also developed cutaneous flushing especially over the chest and face. Heart rate increased up to 140 /minute with ECG showing normal configuration of complexes. Blood pressure with sphygmomanometer was recorded to be $40 \mathrm{~mm}$ of $\mathrm{Hg}$ with very feeble pulse. In the mean time patient was intubated with cuffed endotracheal tube of internal diameter $8.0 \mathrm{~mm}$ facilitated with Succinylcholine 100 mg. Normal saline was infused rapidly from another IV access, obtained with $16 \mathrm{Gz}$ canula in contralateral hand. Chest auscultation was normal with normal vesicular breath sounds bilaterally. After two liters of crystalloid, systolic blood pressure was still $40 \mathrm{~mm}$ of $\mathrm{Hg}$. The patient was then kept in Trendelenberg position. $5 \mathrm{ml}$ of Adrenaline $(1: 10,000)$ was given. Systolic blood pressure was $60 \mathrm{~mm}$ of $\mathrm{Hg}$ after few minutes but failed to rise further. Oxygen saturation was $90 \%$ at this moment. Hemaccele $500 \mathrm{ml}$ was given rapidly. Second dose of $5 \mathrm{ml}$ of the adrenaline was given. The blood pressure recorded was $90 / 50 \mathrm{~mm}$ of $\mathrm{Hg}$, heart rate decreased to $110 /$ minute and oxygen saturation was $100 \%$. Hypotensive episode lasted for about 15 mins. Midazolam $2 \mathrm{mg}$ was given intravenously. Anesthesia was maintained with oxygen, halothane and vecuronium $4 \mathrm{mg}$ was given as muscle relaxant. Surgery was started which lasted for 25 minutes. The patient was extubated awake in the operation theatre after reversal of muscle relaxant with neostigmine $2.5 \mathrm{mg}$ and atropine $1.2 \mathrm{mg}$. The patient was then transferred to post operative ward where he was observed for 24 hours. He had normal vitals. Urine output was $1900 \mathrm{ml}$ in 24 hours. He was transferred to ward the next day and discharged after seven days.

Allergic tests (Intradermal test, Prick test) to thiopentone could not be done as the follow up of the patient was lost.

\section{DISCUSSION}

The incidence of anaphylactic reactions to anesthetic is estimated to be from $1: 5000$ to $1: 30,000$. Anaphylactic reactions to thiopental occur in approximately 1 in 20,000 to 30,000 cases. ${ }^{1}$ Non immunologically mediated anaphylactoid reactions are more common than the true anaphylaxis. Anaphylactoid reactions resemble anaphylaxis but do not depend upon IgE antibody interaction with antigen. Although the mechanisms are different, anaphylactoid reactions and anaphylaxis can be clinically indistinguishable but equally life threatening.
In this patient the cardiovascular collapse following thiopental reaction can be termed anaphylactoid reaction as the patient was not exposed to the drug in the past. Confirmation of the diagnosis of the anaphylactoid reaction can be made by serum histamine level $(0-6 \mathrm{nmol} / \mathrm{L})$, neutral protease, tryptase levels and serum complement levels. ${ }^{2}$ In vivo, histamine is stored in mast cells and basophils, while neutral protease, tryptase (tryptases) is located in significant amount in only secretory granules of mast cells. Therefore increase in serum histamine level may reflect the degranulation of either mast cells or basophils whereas increase in tryptase levels shows degranulations of mast cells. ${ }^{3}$ Hirshman et al. demonstrated in vitro thiopental induced histamine release from the leucocytes of a patient who developed cardiovascular collapse and cutaneous flushing but had no evidence of an immunoglobulin E mediated hypersensitivity with thiopental administration.

The clinical manifestations of a histaminoid reaction are many which involve multisystems of the body. Ninety percent of reactions involve cardiovascular collapse and in $10 \%$ may be the only presenting feature. ${ }^{4,5}$ Supraventricular tachyarrhythmias are common which occur in $80 \%$ of the patients. Incidence of cardiac arrest although rare can occur in $11 \%$ of patients. Respiratory system was not involved in this patient, however bronchospasm can occur in up to $50 \%$ of the patients which may be either transient or severe ${ }^{4,6}$ and in $3 \%$ of patients may be the only presenting feature. Upper airway edema may be present in $12 \%$ of the patients that can be the immediate life threatening problem. Our patient also developed stridor after extubation which gave the suspicion of upper airway oedema for which hydrocortisone was given. Generalized erythema or flushing is the most common skin manifestation, which was also evident in our patient. The characteristic erythematous wheals are easy to recognise but are uncommon. Conjunctival inflammation, as in this patient, is also one of the manifestation which may be assosciated with conjunctival swelling, eyelid oedema and/or lacrimation. Hematemesis, melaena and/or bloody diarrhoea may also be evident that indicates the involvement of gastro intestinal system, ${ }^{4,6}$ which was not seen in this patient. Disseminated intravascular coagulation though rare, may be one of the sequel.

Anesthetic misadventure may also mimic as a histaminoid reaction. It is important to differentiate a true reaction from other conditions causing bronchospasm, hypotension or both, during anaesthesia. Few of the conditions are inhalation of gastric contents, pre existing 
reactive airway disorder relative or absolute overdose of anesthetic agents, hypovolemia, arrhythmias, myocardial infarction.

The mainstay of the treatment of anaphylactoid reaction is $100 \%$ oxygenation, fluid resuscitation and adrenaline. Adrenaline is the drug of choice for hypotension, bronchospasm and angio-oedema. Rapid fluid administration is important from a wide bore canula, which may range to up to 2-3 liters within few minutes. The choice of fluids among crystalloid or colloids still remains in debate, however neither of the fluid has been failed to be superior over one another. ${ }^{7}$ Colloid may be administered if 1.5 to 2 liters of crystalloid fails to raise blood pressure. However one case report with comparison of two cases has found colloid to be superior. ${ }^{2}$ Adrenaline is given at a dose of $0.1-0.5 \mathrm{mg}$ intravenously depending upon the severity of the condition. It can be repeated at 5 mins interval. ${ }^{4}$ Ventricular arrhythmias can occur in patients receiving halothane as inhalational agent after intravenous adrenaline. So halothane should be discontinued, after which other inhalational agent is used if available.
Benzodiazepine (midazolam) can be used as in this case after discontinuation of halothane. Bronchospasm, if occurred, can be relieved by inhalational anesthetic (halothane), salbutamol inhaler or nebuliser via endotracheal tube or anesthetic circuit, inhalational anticholinergic agents (ipratropium bromide), nebulised adrenaline or intravenous aminophylline $(5 \mathrm{mg} / \mathrm{kg})$ or intravenous ketamine. Role of antihistamine in established anaphylactic reaction is controversial. ${ }^{4,6}$

\section{CONCLUSION}

Anaphylactic reaction to thiopental, though rare, may occur with full blown multisystem manifestation or only cardiovascular collapse. Adrenaline is the drug of choice for hypotension, bronchospasm and angio edema as well. Fluid resuscitation is equally important. Here a case of successful management of anaphylactic reaction after thiopental reaction is presented. However serum histamine level or tryptase levels could not be done as the facility is not available, which made us unable to quote whether the source of histamine is mast cells or basophils or both.

\section{REFERENCES}

1. Clark RSJ. Adverse effects of intravenously administered drugs used in anesthesia practice. Drug 1988;22: 26-41.

2. Sprung, Juraj, Schoenwald Peter K, et al. Cardiovascular collapse resulting from Thiopental induced Histamine Release. Anesthesiology 1997;86:1006-7.

3. Schwartz LB, Metclafe DD, Miller JS, Earl H, Sullivan T. Tryptase levels as an indicator of mast cell activation in systemic anaphylaxis and mastocytosis. N Eng J Med 1987;316:1622-6.

4. McKinnon RP, Wildsmith JAW. Histaminoid reactions in anesthesia. British Journal of Anesthesia 1995;74:217-228.

5. Fisher MM, Baldo BA. The incidence and clinical features of anaphylactic reactions during anaesthesia in Australia. Annales Francaises D'Anesthesie et de Reanimation 1993;12:97-104.

6. Fisher MM, Baldo BA. Acute anaphylactic reactions. Medical Journal of Australia 1988; 149:34-38.

7. Fisher MM, Dicks I. Volume replacement in acute anaphylactoid reactions. Anaesthesia and Intensive Care 1979; 7:375-376. 